\title{
Saturation properties of four-wave mixing between short optical pulses in semiconductor optical amplifiers
}

Mørk, Jesper; Mecozzi, A.; Diez, S.

Published in:

Proceedings of CLEO'99

Link to article, DOI:

10.1109/CLEO.1999.834103

Publication date:

1999

Document Version

Publisher's PDF, also known as Version of record

Link back to DTU Orbit

Citation (APA):

Mørk, J., Mecozzi, A., \& Diez, S. (1999). Saturation properties of four-wave mixing between short optical pulses in semiconductor optical amplifiers. In Proceedings of CLEO'99 (pp. 217-218). IEEE.

https://doi.org/10.1109/CLEO.1999.834103

\section{General rights}

Copyright and moral rights for the publications made accessible in the public portal are retained by the authors and/or other copyright owners and it is a condition of accessing publications that users recognise and abide by the legal requirements associated with these rights.

- Users may download and print one copy of any publication from the public portal for the purpose of private study or research.

- You may not further distribute the material or use it for any profit-making activity or commercial gain

- You may freely distribute the URL identifying the publication in the public portal

If you believe that this document breaches copyright please contact us providing details, and we will remove access to the work immediately and investigate your claim. 

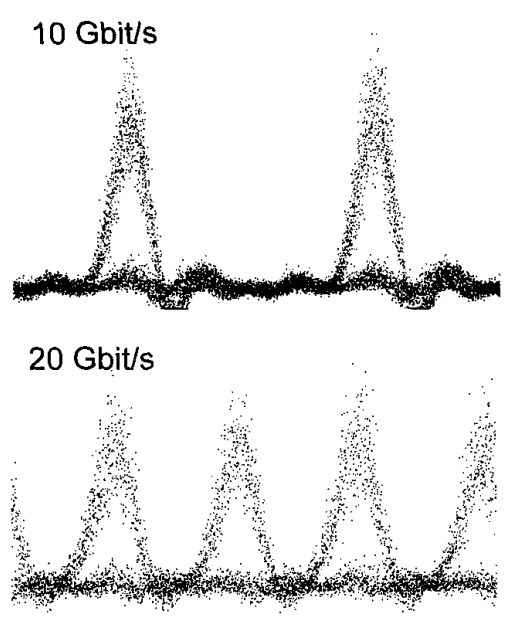

CTuW3 Fig. 3. Experimental measured eyediagrams for a bi-directional transmitted signal at 10 and $20 \mathrm{Gbit} / \mathrm{s}$, respectively.

directional MSSI swapping at 10 and $20 \mathrm{Gbit} / \mathrm{s}$ are shown in Fig. 3. The coupled CW pump power in the experiment was 6 to $7 \mathrm{dBm}$. The coupled signal power was around -6 to $-7 \mathrm{dBm}$. The difference in $\mathrm{CW}$ pump power for equal FWM output power for up- and down-conversion was $2-3 \mathrm{~dB}$. These values are in good agreement with the model predictions.

In conclusion, large signal simulations of a novel scheme of bi-directional FWM are shown to be in good agreement with experiments and identify critical aspects of the system performance.

1. D.D. Marcenac et al., Electron. Lett. 33, 879 (1997).

2. A. Buxens et al., ECOC'98 3, 95 (1998).

3. J. Mark et al., Appl. Phys. Lett. 61, 2281 (1992).

\section{cruW4}

5:15 pm

Demonstration of four wave mixing in an integrated pump laser and semiconductor optical amplifier for midspan spectral inversion dispersion compensation

M.F.C. Stephens, K.A. Williams, R.V. Penty, I.H. White, Department of Electrical and Electronic Engineering, University of Bristol, Queens Building, University Walk, Bristol, United Kingdom BS8 1TR; E-mail: M.F.C.Stephens@bristol.ac.uk

Non-degenerate four wave mixing (NDFWM) has been studied extensively in recent years as both a method for compensating against the dispersion of standard single mode optical fibre via mid-span spectral inversion (MSSI) ${ }^{1}$ and as a transparent wavelength conversion mechanism. NDFWM has been reported in many devices, including semiconductor optical amplifiers (SOAs), distributed feedback (DFB) lasers, nonlinear waveguides and optical fibre itself. ${ }^{2}$ SOAs are promising devices for NDFWM due to their small size, large gain bandwidth and high speed $(>100 \mathrm{~Gb} / \mathrm{s}) .^{3}$ However, an external pump laser and EDFA

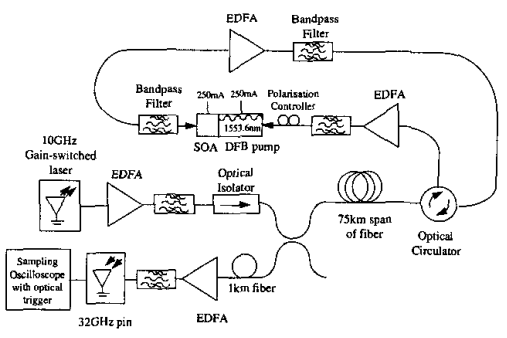

CTuW4 Fig. 1. Schematic of the experimental MSSI arrangement.

a)

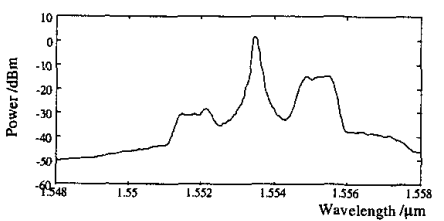

b)

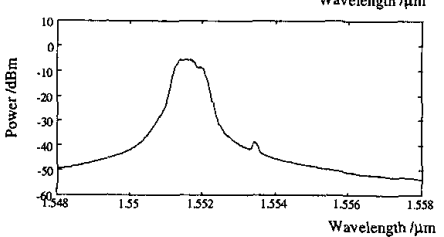

CTuW4 Fig. 2. (a) Spectrum taken directly after the integrated DFB pump/SOA, clearly showing the newly generated phase conjugate at $1552 \mathrm{~nm}$. (b) The phase conjugate signal after filtering and amplification.

are required which adds both cost and complexity to the system. Recently, an integrated DFB laser and SOA has been reported for wavelength conversion under static conditions. ${ }^{3}$ Here, for the first time we believe, the use of a similar device for dynamic MSSI dispersion compensation is demonstrated.

The experimental arrangement is depicted in Fig. 1.

A $10 \mathrm{GHz}$ train of 18 ps pulses is generated by gain-switching a $1555.2 \mathrm{~nm}$ DFB laser. These pulses are amplified by an EDFA and injected into a $75 \mathrm{~km}$ span of standard fibre. The output of the span is passed via an optical circulator into another EDFA and filter before injection into the DFB laser section of the phase conjugator. A polarisation controller is used to match the polarisation of the incoming signal to that of the DFB pump beam. The DFB pump wavelength is at $1553.6 \mathrm{~nm}$ and has a linewidth of $\sim 2 \mathrm{MHz}$ when both it and the SOA are biased at $250 \mathrm{~mA}$. The newly generated phase conjugate signal at $1552 \mathrm{~nm}$ is filtered to remove the pump and original signal before being amplified, filtered again and transmitted back over the span. The received signal is analysed after traversing an additional $1 \mathrm{~km}$ of standard fibre, which compensates for the dispersion induced by the wavelength difference on the two spans. The pulses are examined using a $32 \mathrm{GHz}$ photodiode and $50 \mathrm{GHz}$ sampling oscilloscope.

The optical spectra produced from the output of the phase conjugator are shown in Fig. 2 before and after filtering. The average injected signal power to the phase conjugator is +3.5

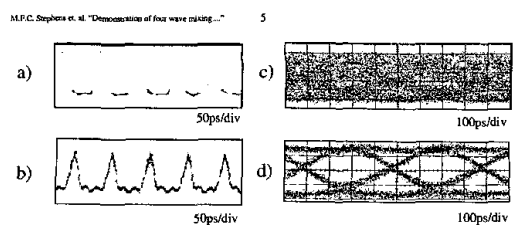

CTuW4 Fig. 3. (a) $10 \mathrm{GHz} 18$ ps input pulses at $1555.2 \mathrm{~nm}$. (b) Received phase conjugate pulse at $1552 \mathrm{~nm}$ after $151 \mathrm{~km}$ of standard fibre. (c) 2.5 $\mathrm{Gb} / \mathrm{s}$ eye with no MSSI at $1555.6 \mathrm{~nm}$. (d) Phase conjugate $2.5 \mathrm{~Gb} / \mathrm{s}$ eye after $151 \mathrm{~km}$.

$\mathrm{dBm}$. After filtering it can be seen that the residual peak pump level is over $30 \mathrm{~dB}$ lower than the phase conjugate.

The back to back and final received pulses are shown in Figs. 3(a), 3(b). It can be seen that there is some degradation of the signal after the $150 \mathrm{~km}$ span. In addition, a highly chirped laser is modulated at $2.5 \mathrm{~Gb} / \mathrm{s}$ and transmitted over the system. The received eye is shown in [Fig. 3(d)] with no significant degradation. Without MSSI, the same eye diagram is completely closed [Fig. 3(c)]

In conclusion, for the first time, an integrated DFB/SOA laser has been shown to operate as an MSSI dispersion compensator via NDFWM with a $10 \mathrm{GHz}$ pulse train and $2.5 \mathrm{~Gb} / \mathrm{s}$ data. Although the former does show some degradation, it is believed that further optimisation of the device (such as reduced DFB linewidth) will lead to far superior performance. A full bit error rate analysis and results at $10 \mathrm{~Gb} / \mathrm{s}$ will be presented at the conference.

1. D.D. Marcenac, D. Nesset, A.E. Kelly, M Brierley, A.D. Ellis, D.G. Moodie, C.W. Ford, Electronics Letters, 1997, 33, No. 10, pp. $879-880$

2. S.J.B. Yoo, IEEE Journal of Lightwave Technology, 1996, 14, No. 6, pp. 955-966.

3. A.E. Kelly, A.D. Ellis, D. Nesset, R. Kashyap, D.G. Moodie, Electronics Letters, 34, No. 20, 1st October 1998, pp. 1955-1956.

4. T. Simoyama, H. Kuwatsuka, B.E. Little, M. Matsuda, Y. Kotaki and H. Ishikawa, 1998 IEEE 16th International Semiconductor Laser Conference, Nara, Japan, paper PD-7, pp. 15-16.

\section{CTuW5}

5:30 pm

Saturation properties of four-wave mixing between short optical pulses in semiconductor optical amplifiers

J. Mørk, A. Mecozzi, ${ }^{*}$ S. Diez, ${ }^{* *}$ Center for Communications, Optics and Materials, The Technical University of Denmark, Bldg. 349, DK-2800 Lyngby, Denmark; E-mail: jm@com.dtu.dk

Four-wave mixing (FWM) between short optical pulses in a semiconductor optical amplifier (SOA) is of considerable interest for performing high-speed optical de-multiplexing or optical sampling. ${ }^{1-3}$ The large gain of the SOA makes efficient FWM over detunings of several nanometers possible. Since FWM relies on physical processes (like carrier density modulation and ultrafast intraband carrier dynamics 


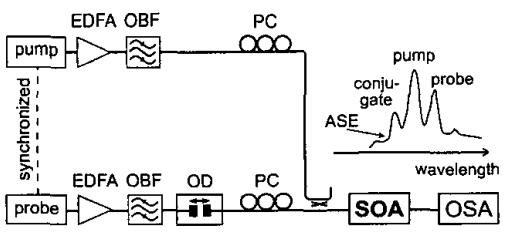

CTuW5 Fig. 1. Experimental setup. Pump $(1540 \mathrm{~nm})$ and probe $(1550 \mathrm{~nm})$ pulses are amplified (EDFA) and band-pass filtered (OBF) before injection into the SOA. An optical delay line (OD) and polarization controllers $(\mathrm{PC})$ are used to align the pulses in time and polarization. The schematic FWM spectrum shows the different wavelength components at the SOA output.

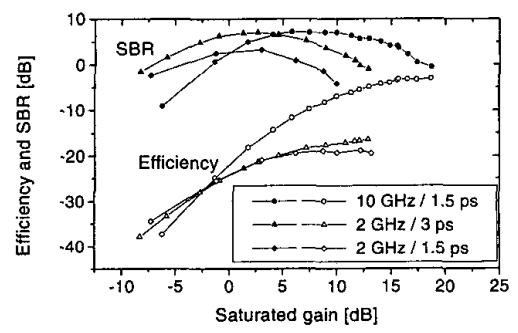

CTuW5 Fig. 2. Experimental results. The average input powers were $3 \mathrm{dBm}$ for the pump and $-7 \mathrm{dBm}$ for the probe. The saturated gain is the fiber-to-fiber gain, including an estimated total coupling loss of $-7 \mathrm{~dB}$.

due to carrier heating and spectral holeburning) that all contribute to gain saturation and therefore counteract the FWM process, the characteristics depend strongly on the operation conditions, i.e., SOA gain, input power, bitrate and pulsewidth.

Figure 1 shows the experimental set-up. Pump and probe pulses are generated using two synchronized external cavity semiconductor modelocked lasers, which can be tuned in pulsewidth and repetition rate. ${ }^{4}$ The pulses are injected, co-polarized and temporally overlapping, into a $980 \mu \mathrm{m}$ long bulk InGaAsP amplifier. The efficiency (conjugate power at output divided by probe input) and the signal-tobackground ratio (SBR) are measured using an optical spectrum analyzer $(1 \mathrm{~nm}$ resolution bandwidth) as a function of the saturated SOA gain. The SOA driving current is varied to adjust to different gain values, which are recorded using a weak $\mathrm{CW}$ beam at the probe wavelength. Measurements for a constant average pump power of $3 \mathrm{dBm}$ (probe is $10 \mathrm{~dB}$ lower) and pulsewidths of $1.5 \mathrm{ps}$ and $3 \mathrm{ps}$ as well as repetition rates of $2 \mathrm{GHz}$ and $10 \mathrm{GHz}$ are shown in Fig. 2. Figure 3 depicts calculated results using the known parameter values from the experiment. The theoretical model incorporates a detailed treatment of propagation effects as well as ultrafast carrier dynamics, but the application of a simple mathematical trick transforms it into a tractable and numerically very effective approach that avoids the solution of partial differential equations. ${ }^{5}$

The calculated results capture all the qualitative effects seen in the experiment. We attribute the quantitative differences between experiment and theory to uncertainties in the parameter values as well as dynamical effects

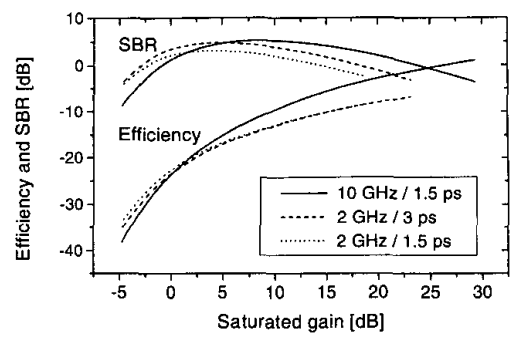

CTuW5 Fig. 3. Calculated results.

due to saturation of the gain coefficients themselves, which is not included in the model.

The existence of a certain gain, or injection current, that optimizes the SBR is special to the interaction between trains of short optical pulses. For CW beams, in contrast, both the efficiency and the SBR increase monotonically with the gain. ${ }^{4}$ The reason for this behavior is that the conjugate pulse experiences the maximum gain saturation since it overlaps with the pump pulse, while the amplified spontaneous emission (ASE) noise has time to recover inbetween the pulses, and therefore experiences a gain which is closer to the unsaturated level. For increasing gain the ASE therefore grows faster than the conjugate energy and a maximum is obtained. Both the calculated and measured data show that the maximum moves to larger gain for the higher repetition rate, but changes only in amplitude for the investigated pulsewidths. We notice that the inclusion of saturation effects due to ultrafast carrier dynamics is absolutely necessary in order to obtain the qualitative dependencies seen in the experiment.

In conclusion, we report the first comparison between theory and experiment on the FWM between trains of short pulses in SOAs. The theory is able to explain all qualitative features seen in the experiment.

${ }^{*}$ Fondazione Ugo Bordoni, via B. Castiglione 59, 00142 Roma, Italy; E-mail: amezozzi@fub.it ${ }^{*}$ Heinrich-Hertz Institut für Nachrichtentechnik, Einsteinufer 37, 10587 Berlin, Germany; E-mail:diez@hhi.de

1. R. Ludwig and G. Raybon, in ECOC'93, pp. 57-60 (1993).

2. M. Shtaif and G. Eisenstein, Appl. Phys. Lett. 66, 1458 (1995).

3. J. Inoue and H. Kawaguchi, IEEE Photon. Technol. Lett. 10, 349 (1998).

4. S. Diez et al., IEEE J. Sel. Topics Quantum Electron, 3, 1131 (1997).

5. A. Mezozzi and J. Mørk, IEEE J. Sel. Topics Quantum Electron. 3, 1190 (1997).

\section{cTuW6}

$5: 45 \mathrm{pm}$

Cross-gain saturation reduction in a double-stage overpumped EDFA with pump re-use

D.A. Carter, Jeff Korn, Lincoln Laboratory, Massachusetts Institute of Technology, 244 Wood Street, Lexington, Massachusetts 02420 9108 USA; E-mail:Dedric@mit.edu korn@LL.mit.edu

Cross-gain saturation in EDFAs gives rise to deleterious effects for WDM, packet TDM net- works, and for secure all-optical networks. ${ }^{1}$ In this paper we make use of the fact that the signal saturation power depends upon the pump saturation level. ${ }^{2}$ Using a short piece of Erbium-doped fiber and utilizing only a fraction of pump light $(\sim 10 \%)$ power excursions are greatly reduced while providing good noise figure and gain. A similar scheme has been analyzed numerically by Willems et al.7 Also demonstrated is pump re-use in a double-stage configuration with inter-stage loss. This architecture could be used to prevent jamming of a frequency-selective switch.'

The single-stage experiment is shown in Fig. 1. Light from a $1545 \mathrm{~nm}$ laser, $\mathrm{Ll}$, is sent through an acousto-optic switch to simulate the addition/dropping of channels. A $1550 \mathrm{~nm}$ probe laser, L2, serves as the surviving channel, and is combined with $\mathrm{L} 1$ via a 50/50 coupler, followed by the overpumped EDFA, consisting of input and output isolators, $13 \mathrm{~m}$ of Lucent's HP-980 erbium-doped fiber, and two WDMs. The pump laser is a fiber-coupled SDL MOPA, providing $450 \mathrm{~mW}$ of co-propagating $984 \mathrm{~nm}$ power. The output of the EDFA is then passed through a 1-nm bandwidth filter and the $1550 \mathrm{~nm}$ signal is displayed on an oscilloscope. The small-signal gain and noise-figure are measured to be $20 \mathrm{~dB}$ and $3.8 \mathrm{~dB}$, respectively, with $\sim 40 \mathrm{~mW}$ of absorbed pump power.

The power of $L 2$ into the EDFA is $-20 \mathrm{dBm}$ and the peak power of $\mathrm{L} 1$ is either $-11,-7$, or $-2.2 \mathrm{dBm}$. This simulates a 9,21 , or 61 $(-20 \mathrm{dBm} /$ channel) channel WDM system where 8,20 , or 60 channels are added/ dropped, respectively, in a purely homogeneous gain medium. Figure 2(a) shows the power excursions were $0.27,0.6$, and $1.5 \mathrm{~dB}$, respectively, for the 3 cases. By comparison, Fig. 2(b) shows the power excursions when a

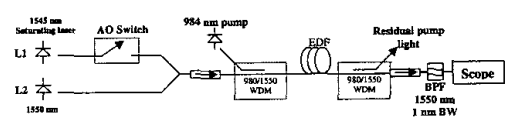

CTuW6 Fig. 1. Experimental setup.
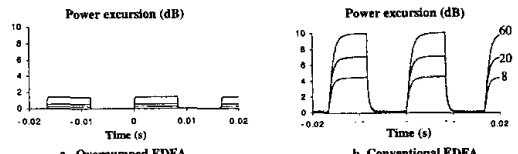

CTuW6 Fig. 2. Power excursion (dB) versus time (ms) for dropping 8,20 , or 60 channels $(-20 \mathrm{dBm} /$ channel).

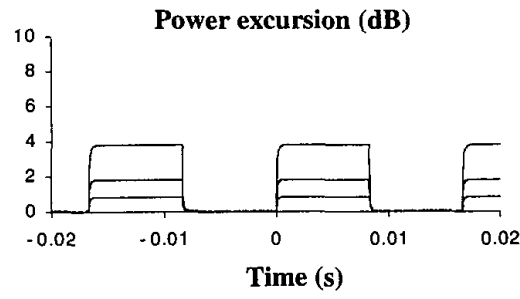

CTuW6 Fig. 3. Power excursion (dB) versus time (ms) for dropping 8,20 , or 60 channels $(-20 \mathrm{dBm} / \mathrm{channel})$ for the double-stage EDFA with $15-\mathrm{dB}$ mid-stage loss and pump re-use. 